\title{
Selective principal component extraction and reconstruction: a novel method for ground based exoplanet spectroscopy
}

\author{
A. Thatte ${ }^{1}$, P. Deroo ${ }^{2}$, and M. R. Swain ${ }^{2}$ \\ 1 Woodruff School of Mechanical Engineering, MRDC Building, Room 4111, Georgia Institute of Technology, Atlanta, \\ Georgia 30332-0405, USA \\ e-mail: azamthatte@gatech.edu \\ 2 Jet Propulsion Laboratory, California Institute of Technology, 4800 Oak Grove Drive, Pasadena, California 91109-8099, USA \\ Received 3 June 2010 / Accepted 12 August 2010
}

\section{ABSTRACT}

\begin{abstract}
Context. Infrared spectroscopy of primary and secondary eclipse events probes the composition of exoplanet atmospheres and, using space telescopes, has detected $\mathrm{H}_{2} \mathrm{O}, \mathrm{CH}_{4}$ and $\mathrm{CO}_{2}$ in three hot Jupiters. However, the available data from space telescopes has limited spectral resolution and does not cover the $2.4-5.2 \mu \mathrm{m}$ spectral region. While large ground based telescopes have the potential to obtain molecular-abundance-grade spectra for many exoplanets, realizing this potential requires retrieving the astrophysical signal in the presence of large Earth-atmospheric and instrument systematic errors.

Aims. Here we report a wavelet-assisted, selective principal component extraction method for ground based retrieval of the dayside spectrum of HD 189733b from data containing systematic errors.

Methods. The method uses singular value decomposition and extracts those critical points of the Rayleigh quotient which correspond to the planet induced signal. The method does not require prior knowledge of the planet spectrum or the physical mechanisms causing systematic errors.

Results. The spectrum obtained with our method is in excellent agreement with space based measurements made with HST and Spitzer and confirms the recent ground based measurements including the strong $\sim 3.3 \mu \mathrm{m}$ emission.
\end{abstract}

Key words. infrared: planetary systems - planets and satellites: atmospheres - techniques: spectroscopic - methods: data analysis methods: numerical

\section{Introduction}

Detection of molecules in exoplanet atmospheres via infrared spectroscopy from space-based telescopes is now routine (Swain et al. 2008; Grillmair et al. 2008; Swain et al. 2009b,a; Tinetti et al. 2010). Recently, Swain et al. (2010) demonstrated molecular spectroscopy of an exoplanet atmosphere with ground-based measurements. With the availability of numerous large ground based telescopes equipped with infrared spectrometers, there is a great potential to obtain a large quantity of "molecularabundance-grade" spectra; realizing this potential requires developing optimal signal extraction algorithms to retrieve the spectral signature of an exoplanet atmosphere in the presence of large Earth-atmospheric and instrument systematic errors. Here we present a method based on principal component analysis (PCA) capable of detecting the exoplanet emission spectrum from the ground. PCA is a well established method with numerous astronomomical applications; it has been used to search for an exoplanet signal in ground-based spectroscopic observations (Brown et al. 2002) and a related method is used in SysRem for exoplanet eclipse detections (Tamuz et al. 2005). We apply our PCA based method to extract the dayside emission spectrum of HD $189733 \mathrm{~b}$ in the $K$ and $L$ bands and we compare the resulting spectrum with previously reported results (Swain et al. 2010), which were obtained using a different method.

\section{Observations and initial calibration}

The spectrum presented here is based on the same observations used in Swain et al. (2010) but analyzed using the selective principal component extraction and reconstruction (SPCER) method described in Sect. 3. A secondary eclipse of HD 189733b was observed on August 11th, 2007 using the SpeX instrument mounted on the NASA Infrared Telescope Facility (IRTF). The spectroscopic time series begins approximately one hour before the onset of ingress and ends approximately one hour after the termination of egress. The details of the observations and the reduction with SpexTool are presented in Swain et al. (2010). The result of the standard SpexTool calibration is a flux density time series with $\sim 4 \%$ variations. We then employ the initial calibration step (normalization \& airmass correction) outlined in Swain et al. (2010). Finally, we separate the exoplanet eclipse astrophysical signal from the residual systematic errors using the SPCER method and thus obtain the exoplanet emission spectrum.

\section{Method}

The astrophysical signal from the exoplanet is present in all spectral channels and we have developed the SPCER method to identify this signal (i.e. the exoplanet eclipse) and separate it from other signal components which are not of astrophysical origin and present in all spectral channels (i.e. systematic errors). The method is based on principal component analysis (PCA), which performs an orthogonal transformation that aligns the transformed axes in the directions of maximum variance. From statistical viewpoint, the eigenvectors of the covariance matrix of a dataset are the critical points of the Rayleigh quotient and when ranked according to the magnitude of the eigenvalues, they represent the axes of maximum variance of that data. To successfully separate non-random signals (systematic errors; the 
astrophysical signal) from random noise, it is desirable that the time series data has a high signal to noise (SNR) ratio. Therefore, we prefilter the time series prior to applying the SPCER method. After prefiltering, we perform singular value decomposition of a set of time series at different wavelengths. This conventiently separates the astrophysical signal from some of the larger systematic errors present in the data. We then reconstruct the astrophysical signal dominated time series and measure the exoplanet emission spectrum. In this section, we outline the methodology. In Sect. 4, we discuss the results of applying this method to the HD 189733b dataset.

\subsection{Prefiltering}

To clean the time series and at the same time retain the dynamic features of the data, we have implemented wavelet transform based adaptive signal extraction. Wavelets are time-localized because the supports of wavelet functions are finite. This makes wavelets excellent for representing discrete events (e.g. abrupt changes linked to outlier points). The wavelet basis functions are constructed from a single function, termed the "Mother Wavelet", $\psi_{0,0}$. The time series is represented as a set of wavelet functions, $\psi_{j, k}$, constructed by a combination of dilation and translation of the mother function: $\psi_{j, k}=2^{j / 2} \psi_{0,0}\left(2^{j} t-k\right)$. Here, we implemented the standard wavelet decompositionthresholding-reconstruction procedure which is widely used in signal and image processing fields (Sidney et al. 1998; Mallat 1989, 1999; Donoho 1995; Donoho \& Johnstone 1995). As mother wavelet, we use the standard Daubechies 3 wavelet (Mallat 1999). We obtain the wavelet decomposition by a multiscale representation and using the transform coefficients $\alpha_{k}^{j}$ ( $k$ th scaling function at $j$ th scale) and $\beta_{k}^{j}$ ( $k$ th wavelet function at $j$ th scale) defined as,

$$
\begin{aligned}
& \alpha_{k}^{j}=\int f(t) \phi\left(2^{j} t-k\right) \mathrm{d} t=\sum_{n} h(n) \alpha_{n+2 k}^{j+1} \\
& \beta_{k}^{j}=\int f(t) \psi_{0,0}\left(2^{j} t-k\right) \mathrm{d} t=\sum_{n} g(n) \alpha_{n+2 k}^{j+1}
\end{aligned}
$$

where the scaling function $\phi$ can be respresented as a signal with a low-pass spectrum and expressed in terms of wavelets (see Mallat 1989); $f(t)$ is the time series for a specific wavelength. $h(n)$ and $g(n)$ represent low pass and high pass filter coefficients respectively and are obtained using Daubechies 3 wavelets in MATLAB. For a detailed description on the theory of wavelet decomposition, the reader is referred to Sidney et al. (1998); Mallat (1989). To produce a smoother estimate and continuous mapping during wavelet shrinkage, we implement a soft thresholding scheme, $\eta_{T}\left(w_{j}\right)=\operatorname{sign}\left(w_{j}\right)\left(\left|w_{j}\right|-T\right)_{+}$, where $w_{j}$ is substituted by the different $\alpha_{k}^{j}$ and $\beta_{k}^{j}$, along with the universal threshold $T=\sigma_{N} \sqrt{2 \ln (N)}$, where $N$ is the current level of wavelet decomposition and $\sigma_{N}$ is the approximation of noise at this level obtained using the robust median estimator. After thresholding, we reconstruct the time series using reconstruction filters and the iterative reconstruction method. Our wavelet based signal extraction algorithm accepts signal $F_{\lambda}(t)$ as input and returns the de-noised signal $F_{\lambda}^{w}(t)$. As a result of this procedure, we get on average a $56 \%$ improvement in the $\frac{\text { standard deviation }}{\text { mean }}$ of the individual time series measurements.

\subsection{Separating multiple patterns in the data}

We start by averaging the time series $F_{\lambda}^{w}(t)$ in the spectral domain (mean of 5 adjacent spectral channels) to reduce the number of PCs and get the time series $\hat{F}_{\lambda}^{w}(t)$. Given $X$, a subset of $\hat{F}_{\lambda}^{w}(t)$ composed of $P$ spectral channels, we center $X$ by subtracting $\bar{X}$, the mean of each column of $X$. Through singular value decomposition we find the eigenvalues $\left(\bar{\lambda}=\left\{\lambda_{1}, \lambda_{2}, \ldots, \lambda_{P}\right\}\right)$ and the eigenvectors (principal components $\overline{\bar{C}}=\left\{C_{1}, C_{2} \ldots C_{P}\right\}$ ) of the covariance matrix of $X$. We then project the centered data onto the principal component axes to get $\Re_{\mathrm{pc}}$, the representation of $\mathrm{X}$ in the principal component space using $\Re_{\mathrm{pc}}=X \otimes \overline{\bar{C}}$ where $\otimes$ denotes matrix multiplication.

For our purposes, we want to decompose the signal present in the ensemble of time series into various components, e.g. flux variation due to secondary eclipse and Earth-atmospheric effects etc. For this, we transform each individual principal component $C_{r}\left(C_{r}=\overline{\bar{C}}(r), r=1,2, \ldots, N\right)$ into the time domain as,

$\overline{\bar{S}}_{r \text { th pc }}=\bar{X}+\overline{\bar{R}}_{\mathrm{pc}}(r) \otimes(\overline{\bar{C}}(r))^{T} \quad$ for $r=1,2, \ldots, N$.

At this point, what we have done is transform our set of time series so that it is expressed in terms of patterns (principal components) that optimally represent the covariance (commonality) of $X$. Each $\overline{\bar{S}}_{r \text { th pc }}$ is such a reconstructed pattern and it shows how the signal would have looked like if it was due to this single PC. We can now determine which PCs represent the eclipse and which represent confusing systematic errors.

\subsection{Extracting the exoplanet spectrum}

To select the principal components corresponding to the eclipse, we calculate the linear correlation coefficient $(C C)$ between each of the individually reconstructed signals $\overline{\bar{S}}_{r \text { th pc }}$ and the expected light curve shape. No prior knowledge on the depth of the eclipse is required for this; only the exoplanet ephemerides(Winn et al. 2007, for HD 189733) is needed. We select a set of principal components capturing the eclipse event using selection criteria described below and reconstruct the data using these components $\left(\overline{\bar{S}}_{\mathrm{Rpc}}\right)$. As a result of this reconstruction, we get an exoplanet eclipse light curve in which the effect of confusing systematics is greatly reduced. For each wavelength channel in $X$, we determine the eclipse depth and the error bar based on the standard deviation of the time series in-eclipse and out-of-eclipse. The eclipse depth for $X$ is the average eclipse depth and the error bar is composed of the error on the eclipse depth for each wavelength channel and the standard deviation of the depths for each wavelength channel in $X$ :

$\sigma_{\text {eclipse depth }}=\sqrt{\left(\frac{\sqrt{\sum_{i=1}^{K}\left(\frac{\sigma_{\text {in }}^{2}}{N_{\text {in }}}+\frac{\sigma_{\text {out }}^{2}}{N_{\text {out }}}\right)}}{\sqrt{K}}\right)^{2}+\sigma_{\text {depths }}^{2}}$

with $\sigma_{\text {in }}$ and $\sigma_{\text {out }}$ the standard deviation of the flux in-eclipse and out-eclipse regions for each wavelength channel in $X ; N_{\text {in }}$ and $N_{\text {out }}$ the number of observations in and out of eclipse; $K$ the number of wavelength channels in $X ; \sigma_{\text {depths }}$ the standard deviation of the eclipse depth for the different wavelength channels in $X$.

The selection criteria to decide whether a principal component captures the exoplanet eclipse event are:

1. $|C C|_{P C, i}>|C C|_{\text {threshold }}$, with $|C C|_{\text {threshold }}=\operatorname{mean}(|C C|)+$ std $\operatorname{dev}(|C C|)$; the correlation coefficient of the PC must be large enough, such that it describes well the eclipse event; 


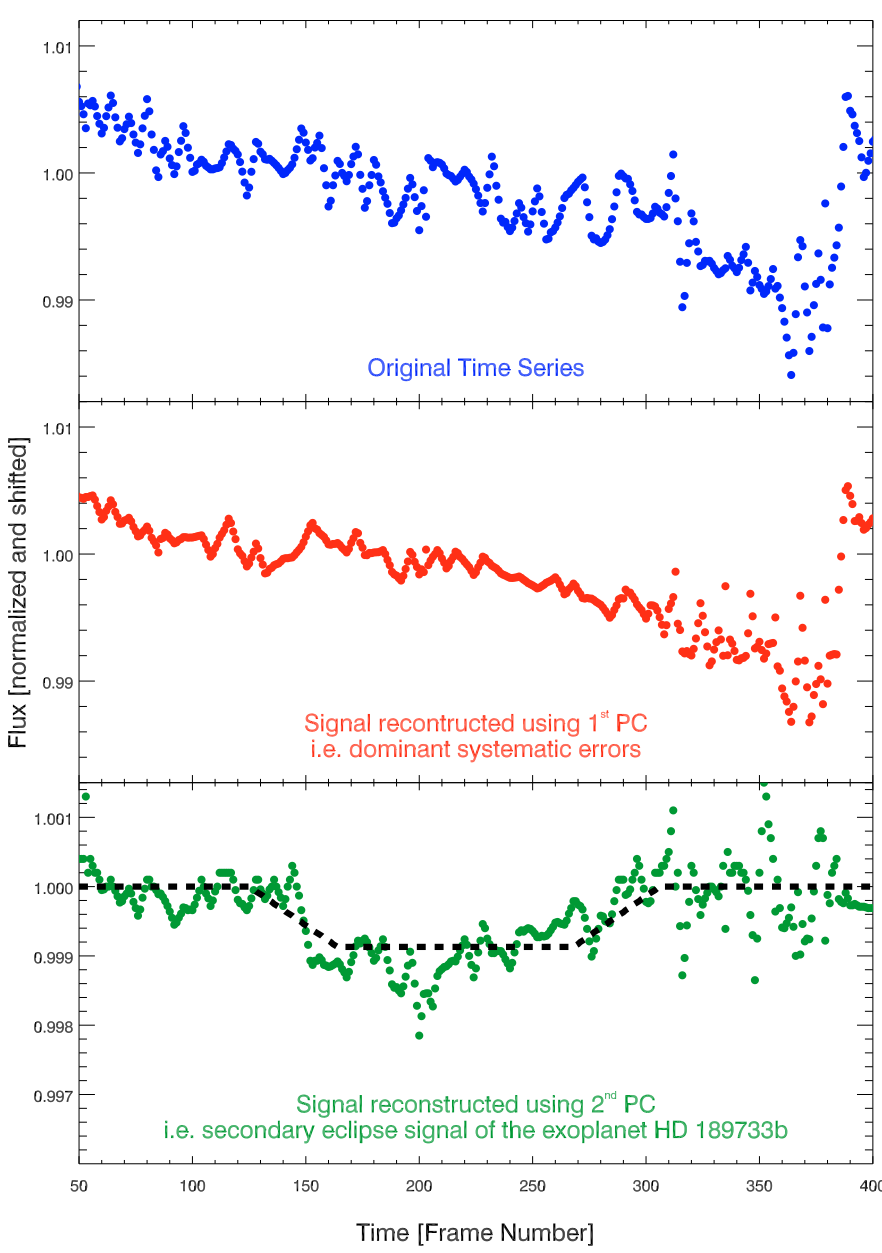

Fig. 1. Separating the astrophysical signal from systematic errors. The different panels depict: top: an original, normalized and airmass corrected time series, middle: signal reconstructed using the first PC, bottom: signal reconstructed using the second PC. In the bottom panel, we show using a black dashed line the secondary eclipse lightcurve overplotted on the reconstructed data. The singular value decomposition of a set of time series at different wavelengths allows us to extract the exoplanet signature, largely free of systematic errors. We show the data at the original, unbinned time sampling.

2. $R_{P C, i}<R_{\text {threshold }}$ with $R_{P C, i}$ the eigenvalue rank of the $i$ th selected PC and $R_{\text {threshold }}$ is the cutoff value for rank. We have chosen $R_{\text {threshold }}=9$ (see later);

3. $\left|R_{P C, i}-R_{P C, i+1}\right|<\Delta_{\text {threshold }} ; \Delta_{\text {threshold }}$ is the cutoff value for the difference in the ranks of the selected PCs. We have chosen $\Delta_{\text {threshold }}=6$ (see later).

If no PC is found under these selection criteria, we assign an upper limit to the eclipse depth, matching the lowest eclipse depth found in the dataset and conclude that we could not find the eclipse for those wavelength channels.

\section{Results and discussion}

We have applied the method outlined above to the spectroscopic time series on the secondary eclipse of HD 189733b observed with the IRTF. We chose to use the same spectral binning as in Swain et al. (2010), such that we can easily compare the results. In the $K$-band and $L$-band, we used 100 and 150 spectral channels respectively to construct $X$. We then processed the data through the different steps outlined above and a sample result of the principal component extraction is shown in Fig. 1. Blue

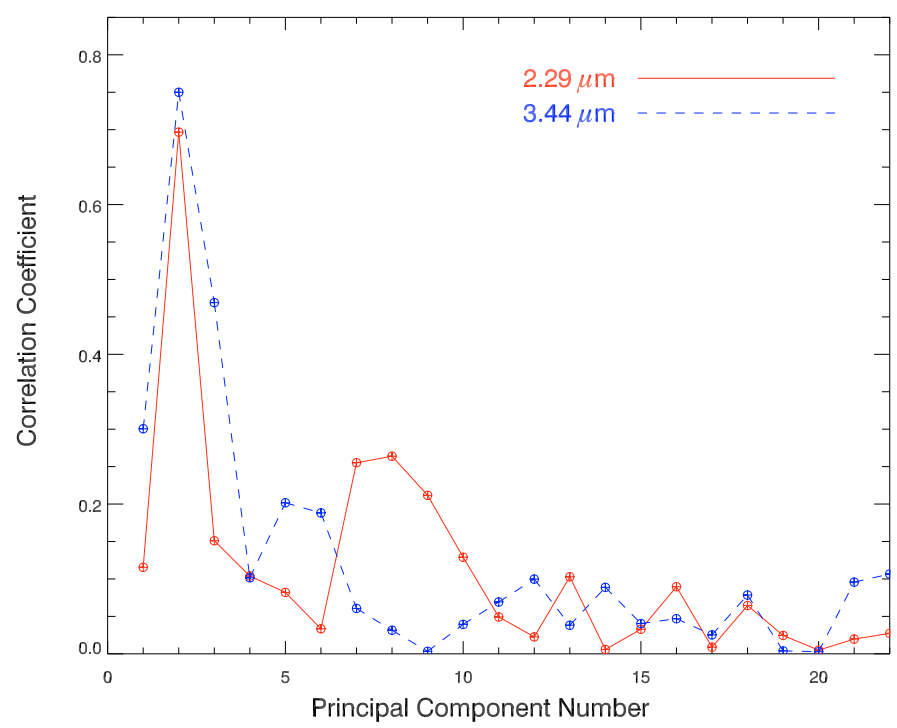

Fig. 2. Selecting the principal components which capture the exoplanet eclipse. The linear correlation coefficients between the PCs and the model eclipse light curve are shown for representative wavelengths for the $K$ and $L$-band. The eclipse is captured by PCs with a high correlation coefficient. The selection of PCs is rather straightforward with very large correlation coefficients being found at low PC numbers.

symbols show the normalized original light curve which is dominated by residual systematic errors. Red and green curves show the signal reconstructed using the first and the second $\mathrm{PC}$ respectively. The model eclipse curve is shown in black. The first PC can be seen to represent much of the systematic errors present in the original signal, while the second PC is clearly the exoplanet eclipse. This demonstrates the potential of SPCER in separating systematic effects for ground based observations and in retrieving the exoplanet signal. It does so without requiring knowledge of the physical mechanism causing the corruption nor requiring prior knowledge on the exoplanet.

To determine the exoplanet spectrum, we have selected $\overline{\bar{S}}_{\mathrm{Rpc}}$ using the criterium outlined above with $R_{\text {threshold }}=9$ and $\Delta_{\text {threshold }}=6$. In Fig. 2, we illustrate the calculated correlation coefficients with the light curve for $K$ and $L$-band wavelengths. It is clearly seen that a PC either matches really well $(C C>0.65)$ or not very well, making the selection rather straightforward and changes in the selection criterium have little influence. The result of the procedure, the exoplanet spectrum of HD 189733b, is shown in Fig. 3. The SPCER method reproduces the measurements by HST (Swain et al. 2009a), the Spitzer photometry (Charbonneau et al. 2008) and those reported in (Swain et al. 2010) extremely well, within the error bars.

A few considerations we would like to mention are:

- Importance of pre-cleaning the data; removing the biggest errors first. The data processing outlined in Swain et al. (2010) includes removal of systematic errors correlated in wavelength and airmass. If this step is not taken, it is still feasible to find the eclipse signal, but at lower eigenvalue rank. For instance, not performing the airmass correction outlined in Swain et al. (2010), shifts the eclipse signal from a typical eigenvalue rank 2 to eigenvalue rank 4.

- Importance of selecting (sometimes) multiple PCs. There is a lack of control in the choice of orthogonal basis functions in the singular value decomposition. This means that the eclipse is not always captured in a single PC, and several PCs (typically two) may be needed to represent the eclipse. When the 

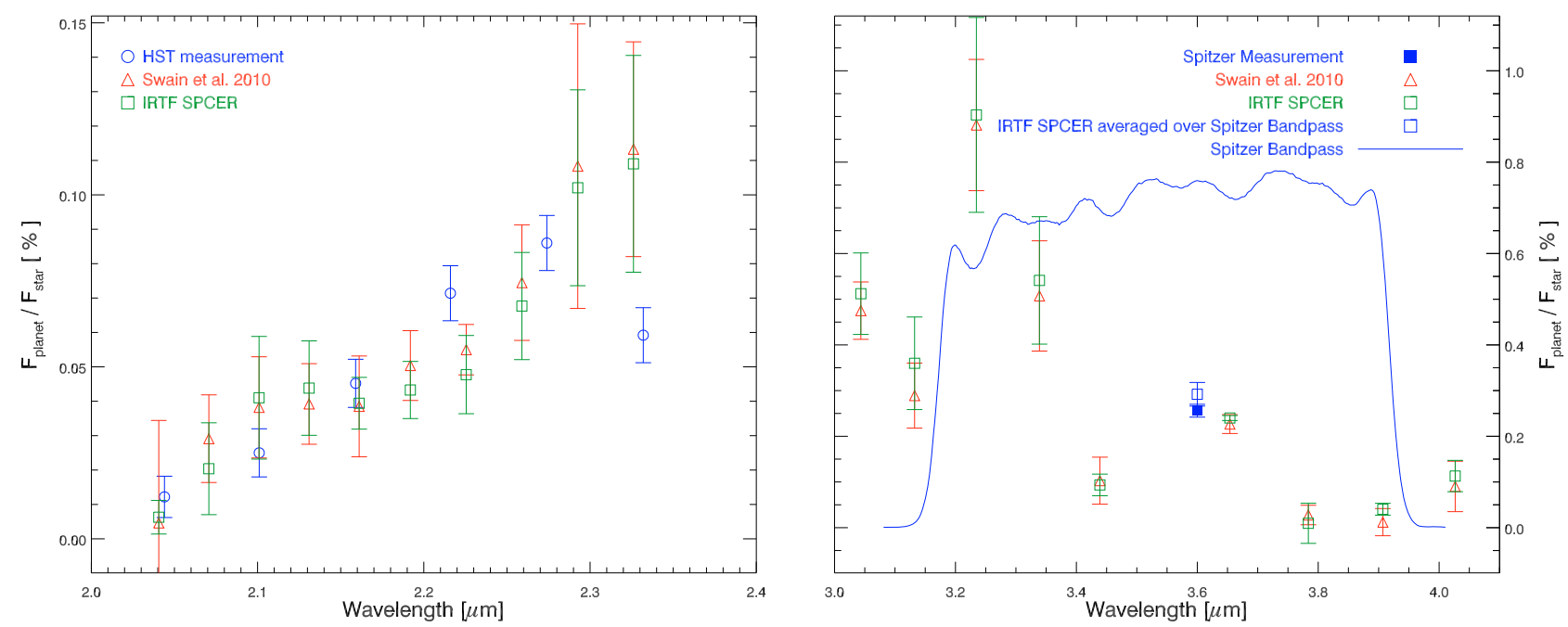

Fig. 3. Dayside emission spectrum. The $K$-band (left) and $L$-band (right) HD 189733b emission spectrum obtained using SPCER is shown using green squares and is compared to the space-based HST spectrum (Swain et al. 2009a) and Spitzer broad-band photometry (Charbonneau et al. 2008) and the ground-based spectrum reported in Swain et al. (2010). We also show the Spitzer passband (blue line) for the $3.6 \mu \mathrm{m}$ photometric point and have averaged the SPCER result over this passband to make comparison easy (blue open symbol). There is excellent agreement between the space and ground-based datasets.

reconstruction is done based solely on one $\mathrm{PC}$, the spectral shape is preserved, but the average eclipse depth can change by up to $\sim 20 \%$.

- Possible improvement by post-processing the PCs. The SPCER method does not necessarily decompose the data entirely into an eclipse and non-eclipse component, because no priors are included (which is also the strength of the method). As such, some residual systematic error can be convolved with the eclipse signal. Simple post-processing, like fitting a polynomial to the out-of-eclipse part of the light curve, can potentially enhance the SNR of the exoplanet spectrum. For this particular dataset, this was not necessary, but might prove advantageous for other datasets.

- Further improvements are likely possible by incorporating the known eclipse shape. In the method presented here, no prior knowledge of the eclipse shape is used during the disentanglement of systematic effects and eclipse signal. Methods that incorporate priors, such as an iterative matched filter, have the potential to improve the method presented here.

- Equal signal to noise ratio for the different wavelength channels is assumed when using PCA. For the dataset analyzed here, the different wavelength channels in each spectral bin $X$ differ only marginally in count rate (the standard deviation of the normalized SNR is on average $4 \%$ and is always less than $13 \%$ for the different wavelength bins $X$ ) and regular PCA is therefore appropriate. If this is not the case, a SysRem type of down-weighting of wavelength channels with low count rates (Tamuz et al. 2005), is needed.

In summary, the coupled wavelet denoising-SPCER method presented here is a new and powerful method for ground based exoplanet calibration. A useful aspect of the method lies in its ability to reject systematic errors without the need for knowledge of the underlying physical mechanism. This gives us the ability to separate the original planetary signal from the relatively large systematic effects. The results of the SPCER-based calibration for the emission spectrum of HD 189733b are in excellent agreement with those obtained with HST (Swain et al. 2009b) and Spitzer (Charbonneau et al. 2008) and confirm the recent ground based measurements (Swain et al. 2010). The strength of SPCER in retrieving the exoplanet spectrum from ground based observations is a proof of concept for its more general application in fields where signal deeply buried under noise must be extracted; the method has applications in a variety of fields including earth and atmospheric sciences, telecommunication systems, measurement instruments, biomedical engineering, optics, image processing and controls, where problems of not having good signal to noise ratio before signal amplification are prevalent or where pattern recognition is critical.

\section{References}

Brown, T. M., Libbrecht, K. G., \& Charbonneau, D. 2002, PASP, 114, 826 Charbonneau, D., Knutson, H. A., Barman, T., et al. 2008, ApJ, 686, 1341 Donoho, D. L. 1995, IEEE Transactions on information theory, 41, 613 Donoho, D. L., \& Johnstone, I. M. 1995, J. Amer. Statist. Assoc., 90, 1200 Grillmair, C. J., Burrows, A., Charbonneau, D., et al. 2008, Nature, 456, 767 Mallat, S. G. 1989, IEEE Transactions on Pattern Analysis and Machine Intelligence, 11, 674

Mallat, S. G. 1999, A Wavelet Tour of Signal Processing, 2nd edn. (Associated Press)

Sidney, B., Ramesh, A. G., \& Haitao, G. 1998, Introduction to Wavelets and Wavelet Transforms: A Primer (Prentice-hall International)

Swain, M. R., Vasisht, G., \& Tinetti, G. 2008, Nature, 452, 329

Swain, M. R., Tinetti, G., Vasisht, G., et al. 2009a, ApJ, 704, 1616

Swain, M. R., Vasisht, G., Tinetti, G., et al. 2009b, ApJ, 690, L114

Swain, M. R., Deroo, P., Griffith, C. A., et al. 2010, Nature, 463, 637

Tamuz, O., Mazeh, T., \& Zucker, S. 2005, MNRAS, 356, 1466

Tinetti, G., Deroo, P., Swain, M. R., et al. 2010, ApJ, 712, L139

Winn, J. N., Holman, M. J., Henry, G. W., et al. 2007, AJ, 133, 1828 